\title{
Long Duration Testing of a Spacesuit Water Membrane Evaporator Prototype
}

Grant C. Bue, Janice Makinen, Marlon Cox, Carly Watts and Colin Campbell

NASA Johnson Space Center, Houston, TX, 77058

Matthew Vogel and Aaron Colunga

Jacobs Engineering, Engineering and Science Contract Group, Houston, TX, 77058

The Spacesuit Water Membrane Evaporator (SWME) is a heat-rejection device that is being developed to perform thermal control for advanced spacesuits. Cooling is achieved by circulating water from the liquid cooling garment (LCG) through hollow fibers (HoFi's), which are small hydrophobic tubes. Liquid water remains within the hydrophobic tubes, but water vapor is exhausted to space, thereby removing heat. A SWME test article was tested over the course of a year, for a total of $\mathbf{1 2 0 0}$ cumulative hours. In order to evaluate SWME tolerance to contamination due to constituents caused by distillation processes, these constituents were allowed to accumulate in the water as evaporation occurred. A test article was tested over the course of a year for a total of 1200 cumulative hours. The heat rejection performance of the SWME degraded significantly--below $700 \mathrm{~W}$, attributable to the accumulation of rust in the circulating loop and biofilm growth. Bubble elimination capability, a feature that was previously proven with SWME, was compromised during the test, most likely due to loss of hydrophobic properties of the hollow fibers. The utilization of water for heat rejection was shown not to be dependent on test article, life cycle, heat rejection rate, or freezing of the membranes. 